\title{
AVALIAÇÃO DE ALGUNS ASPECTOS DA AQUISIÇÃO E DESENVOLVIMENTO DA LINGUAGEM DE CRIANÇAS NASCIDAS PRÉ-TERMO
}

\author{
Mara Rita Pereira1, Carolina Araújo Rodrigues Funayama²
}

\begin{abstract}
RESUMO - A correção da idade para avaliação motora de nascidos pré-termo tem sido consenso, o que não ocorre em outros domínios do desenvolvimento. Este estudo comparou indicadores da aquisição e desenvolvimento da linguagem, considerando-se as idades cronológica e corrigida. Foram acompanhadas por 1 a 15 meses 20 crianças hígidas nascidas entre 28 e 36 semanas (mediana 32s), com 800g a 2380g (mediana $1590 \mathrm{~g}$ ), sendo 9 adequado para a idade gestacional (AIG) e 11 pequenas para a idade gestacional (PIG). A referência de normalidade foi o roteiro de Costa et al. (1992), que contém cinco níveis de linguagem. Quanto aos comportamentos receptivos, já considerando-se a idade cronológica, houve desempenho normal em todos os níveis, exceto no nível I (0-3 meses). Em relação à linguagem expressiva, considerando-se a idade cronológica, das 50 avaliações, 6 (12\%) foram normais. Com a correção da idade, em 16 avaliações (40\%) as crianças adequaram-se ao nível esperado, sendo mais freqüente a adequação aos 6 e 12 meses. Considerando-se a idade cronológica, houve maior número de AIG com desempenho normal $(p<0,05)$. Com o uso da metodologia de Costa, não foi necessário corrigir a idade para avaliação da linguagem receptiva e, para a expressiva, a freqüência maior de resultados normais nas idades corrigidas para 6 e 12 meses sugere intensificação de vigilância nestas idades.
\end{abstract}

PALAVRAS-CHAVE: desenvolvimento, linguagem, pré-termo, idade corrigida.

\begin{abstract}
Evaluation of some aspects of the acquisition and development of language in pre-term born children

ABSTRACT - The correction of the age of pre-term infants for the motor evaluation has been the accepted practice but it has not been clear in other areas. This study compared indicators of the acquisition and development of language, considering corrected and chronological ages. Twenty healthy infants born between the $28^{\text {th }}$ and $36^{\text {th }}$ week of gestation (median 32 weeks), weighing $800 \mathrm{~g}$ to $2380 \mathrm{~g}$ (median 1590g), 9 AGA and 11 SGA, were followed up to 15 months age. As a reference for normality, evaluation of Costa et al. (1992) was used, which groups predictable behavior in 5 levels. For receptive language, considering the chronological age, normal performance occurred at all levels except for Level I (0-3 months). For expressive language, considering the chronological age, $6(12 \%)$ of the 50 evaluations showed normal performance. With their age corrected, in 16 evaluations (40\%) the infants achieved the expected level, mainly at 6 and 12 months age. On the whole, for the chronological age, there was a larger number of AGA with normal performance $(p<0.05)$. We conclude that with the use of the Costa method, it was unnecessary to correct the age for receptive language evaluation, and that, for the expressive, the high frequency of normal results at the corrected ages for 6 and 12 months, suggests that these ages constitute periods of intensification of vigilance.
\end{abstract}

KEY WORDS: development, language, pre-term, corrected age.

Com a evolução da assistência em unidades de terapia intensiva neonatal, a taxa de sobrevivência dos recém-nascidos considerados de risco ao nascimento tem aumentado; por outro lado, essas crianças estão mais propensas a apresentar altera- ções ou desvios em suas aquisições e desenvolvimento da linguagem, fala, motor, auditivo e cognitivo, o que vem despertando a atenção dos pesquisadores.

Os primeiros estudos relacionados à necessidade

Departamento de Neurologia, Faculdade de Medicina de Ribeirão Preto da Universidade de São Paulo, Ribeirão Preto SP, Brasil (USP/Ribeirão Preto): ${ }^{1}$ Fonoaudióloga, Especialista em Distúrbios da Comunicação Humana pela Universidade Federal de São Paulo (UNIFESP), Mestre em Neurociência, Área de Concentração: Neurologia (USP/Ribeirão Preto); ${ }^{2}$ Neuropediatra, Professora Doutora da Faculdade de Medicina de Ribeirão Preto(USP/Ribeirão Preto), responsável pelo Serviço de Neurologia Infantil do Hospital das Clínicas (Ribeirão Preto)

Recebido 28 Novembro 2003, recebido na forma final 25 Fevereiro 2004. Aceito 26 Março 2004.

Dra. Mara Rita Pereira Jorge - Rua Santos Pereira 581 - 14401-130 Franca SP - Brasil. E-mail: marapereira@netsite.com.br 
da correção da idade para avaliação do desenvolvimento do pré-termo iniciaram-se com Babson em $1970^{1}$ na área de crescimento. Posteriormente, estendeu-se o enfoque para o valor da correção da idade para avaliações do desenvolvimento ${ }^{2-4}$, sendo, a partir da década de 1980, recomendada para a avaliação neuromotora ${ }^{5,6}$. Siege $\mathrm{l}^{2}$ e Ungerer e Sigman ${ }^{3}$ foram os primeiros a estudar mais extensivamente a linguagem: comparando idade cronológica à corrigida e centralizaram o interesse na investigação do momento em que o pré-termo se iguala à criança nascida a termo, ponderando sobre os riscos e benefícios da correção, como a maioria dos estudos que se seguiram. A inclusão nos estudos específicos sobre linguagem, de variáveis como a condição de pequenos para a idade gestacional (PIG) e adequado para a idade gestacional (AIG), surgiu mais recentemente e despertou interesse, dadas as condições desfavoráveis relacionadas ao PIG, já bem estabelecidas ${ }^{7-10}$.

Utilizando como parâmetro comportamentos receptivos e expressivos de linguagem, propostos por roteiro publicado em nosso meio ${ }^{11}$, a presente pesquisa tem por objetivo comparar indicadores da aquisição e desenvolvimento da linguagem receptiva e expressiva, de crianças nascidas pré-termo, sem sinais de lesão neurológica e com acuidade auditiva normal, considerando-se a idade cronológica e corrigida, e a condição de nascimento AIG ou PIG.

\section{MÉTODO}

Foram avaliadas 69 crianças de 2 a 15 meses de idade que nasceram pré-termo, e entre estes, posteriormente foram selecionados para este estudo 20 considerados hígidos com idade gestacional (IG) de 28 a 35 semanas, mediana 32 semanas, sendo 12 crianças do sexo masculino e 8 do feminino. $O$ peso ao nascimento variou entre $800 \mathrm{~g}$ e $2380 \mathrm{~g}$, média de $1590 \mathrm{~g}$. Onze dessas 20 crianças eram PIG, 9 AIG, e nenhuma grande para a idade gestacional, de acordo com a avaliação de rotina do berçário do Hospital das Clínicas de Ribeirão Preto (HCRP), que utiliza os métodos de Capurro ${ }^{12}$ e Ballard ${ }^{13,14}$ para o cálculo da idade gestacional ao nascimento, e as curvas de Sala ${ }^{15}$ para a relação peso e idade gestacional. Apenas uma das crianças convivia somente com a mãe, em uma família com cinco pessoas, entre tios e avós. As demais conviviam com os pais e irmãos. Todos os pais e mães eram alfabetizados, variando de primeira série incompleta à oi-

Tabela 1. Sinopse da avaliação fonoaudiológica.

\begin{tabular}{|c|c|c|}
\hline Comportamentos & & \\
\hline \multicolumn{3}{|l|}{ A - Pré-lingüístico } \\
\hline Nível I & Emissão & Produz sons (por exemplo, "guturais") \\
\hline (0 - 3 meses) & Recepção & Aquieta-se ao escutar a voz da mãe/reage à voz da mãe \\
\hline \multicolumn{3}{|l|}{ Nível II } \\
\hline \multirow[t]{3}{*}{ (4 -6 meses) } & Emissão & Vocaliza \\
\hline & & Reconhece a voz da mãe \\
\hline & Recepção & Atende quando chamado \\
\hline Nível III & Emissão & Emite sílabas repetidas \\
\hline (7 - 9 meses) & Recepção & Reconhece o nome \\
\hline \multicolumn{3}{|l|}{ B - Lingüístico } \\
\hline \multirow{6}{*}{$\begin{array}{l}\text { Nível IV } \\
(10-12 \text { meses })\end{array}$} & Emissão & Emite sílabas repetidas \\
\hline & & Emite de duas a três palavras com significado \\
\hline & Recepção & Compreende ordens sem gesto \\
\hline & & "Dá tchau", "dá beijo" \\
\hline & & Compreende situações familiares como: \\
\hline & & "papai chegou!" "vamos passear!" \\
\hline \multirow{3}{*}{$\begin{array}{l}\text { Nível V } \\
\text { (13 - } 15 \text { meses) }\end{array}$} & Emissão & Produz onomatopéia \\
\hline & & Nomeia objetos, pessoas e situações \\
\hline & Recepção & $\begin{array}{l}\text { Reconhece ordens simples: "cadê chupeta?", } \\
\text { "cadê a mamãe?", "cadê o nenê?" }\end{array}$ \\
\hline
\end{tabular}


tava série, com maior freqüência entre segunda e quarta séries do primeiro grau. A renda mensal de cada família foi em torno de dois a três salários mínimos vigentes na época. As crianças foram acompanhadas desde o nascimento até a alta hospitalar e, posteriormente, em retornos mensais pelo Serviço de Neurologia Infantil do Departamento de Neurologia, Psiquiatria e Psicologia Médica da Faculdade de Medicina de Ribeirão Preto. As avaliações de linguagem foram realizadas subseqüentemente às da Neurologia Infantil após a alta hospitalar.

A correção da idade cronológica foi realizada de acordo com a proposta de Babson', subtraindo-se da idade cronológica o período decorrido da idade gestacional ao nascimento e quarenta semanas, que é a média do termo gestacional, considerado entre 38 e 42 semanas.

As avaliações foram consideradas cegas em função da examinadora não ter tido o conhecimento prévio de que, entre as 69 crianças acompanhadas, seriam selecionadas pela neuropediatra responsável 20 crianças com ultra-sonografias de crânio normais no período perinatal e exames neurológicos normais desde o nascimento até a última avaliação aos 15 meses de idade cronológica. As crianças foram previamente avaliadas quanto à percepção de sons instrumentais, em protocolo de detecção precoce de deficiência auditiva, tendo sido consideradas sem alterações pelo Setor de Fonoaudiologia do HCRP.

Para avaliação do desenvolvimento da linguagem, foi utilizado o roteiro de avaliação fonoaudiológica, baseado no desenvolvimento normal da criança proposto por Costa et al. ${ }^{11}$. Foi utilizado deste roteiro o item 4 "caracterização dos aspectos comportamentais", sub-item 4.3 - Linguagem. Foram selecionados alguns dos comportamentos receptivos e expressivos pré-lingüísticos e lingüísticos de zero até 15 meses de idade, dentre os vários comportamentos que o roteiro propõe, dando-se preferência aos comportamentos, com os quais os pais habitualmente demonstram maior familiaridade, dentro de cada faixa etária, representadas em níveis I, II, III, IV, $\mathrm{V}$, (Tabela 1 ). Os desempenhos foram considerados normais se preenchessem pelo menos um item dos investigados, em cada nível, para cada tipo de linguagem, receptiva ou expressiva.

As idades foram consideradas em meses completos e divididas em idade inferior, mediana e superior (Nível
I - 1, 2, 3 meses; Nível II - 4,5,6 meses; Nível III - 7,8,9 meses; Nível IV - 10,11,12 meses e Nível V - 13,14 e 15 meses).

Para a análise dos resultados, o desempenho observado nas idades cronológicas foi comparado aos dos níveis propostos por Costa et al. ${ }^{11}$, conforme a Tabela 1 (considerados esperados), e depois, novamente confrontados, após a correção da idade.

Os casos com idade cronológica e corrigida no mesmo nível foram excluídos. Nos casos com duas avaliações no mesmo nível, tanto para a idade cronológica como para a corrigida, foi selecionada a última avaliação do período.

A sala continha uma mesa, três cadeiras, uma maca e brinquedos apropriados para a faixa etária da criança. Inicialmente, foi realizada a anamnese e entrevista dirigida com a mãe, e em seguida, a criança foi avaliada em uma variedade de situações lúdicas, conforme a oportunidade se apresentava. Foram utilizados brinquedos apropriados a cada faixa etária, sendo os mesmos para todas as crianças. Durante toda a realização da anamnese e avaliação, a mãe, o pai, e inclusive irmãos, quando possível, estiveram presentes e participantes na sala. Foram consideradas como condições adequadas ao exame, a criança estar acordada, não manifestando desconforto. O tempo decorrido entre anamnese e observação direta realizada pela fonoaudióloga foi de uma hora.

Ao registrar as manifestações do desenvolvimento da linguagem individualmente, procurou-se associar todos os comportamentos da criança referidos pela mãe na entrevista dirigida e os dados da observação direta realizada pela fonoaudióloga.

Para o tratamento estatístico foram utilizados o teste não paramétrico de correlação entre proporções e o teste exato de Fisher. Considerou-se o nível de significância de $5 \%$.

Este estudo foi aprovado pelo Comitê de Ética do HCRP, processo $n^{\circ} 5008 / 2003$, e realizado após assinatura, pelas mães, do termo de consentimento pós-informado.

\section{RESULTADOS}

Foram realizadas 50 avaliações em todo o período de estudo (Tabela 2). Para a linguagem receptiva, considerando-se a idade cronológica de zero a 3 meses, entre as 10 crianças avaliadas, 8 não cor-

Tabela 2. Linguagem expressiva: mudança de nível.

\begin{tabular}{lccccc}
\hline & \multicolumn{5}{c}{ N $^{\circ}$ de correções de idade } \\
\cline { 2 - 6 } & II para I & III para II & IV para III & V para IV & total \\
\hline Normalização & 0 & 9 & 3 & 4 & 16 \\
Ambas atrasadas & 8 & 2 & 8 & 1 & 19 \\
Ambas normais & 2 & 1 & 0 & 2 & 5 \\
Total & 10 & 12 & 11 & 7 & 40 \\
\hline
\end{tabular}


Tabela 3. Linguagem expressiva: distribuição do número de avaliações de acordo com a idade.

\begin{tabular}{|c|c|c|c|c|c|c|c|c|c|}
\hline \multicolumn{5}{|c|}{ Cronológica } & \multicolumn{5}{|c|}{ Corrigida } \\
\hline Nível & $\mathrm{N}$ & Inferior & Mediana & Superior & Nível & $\mathrm{N}$ & Inferior & Mediana & Superior \\
\hline I & 10 & 0 & $8(1)^{*}$ & 2 & I & 10 & 1 & $4(1)$ & $5(1)$ \\
\hline II & 10 & $6(1)$ & $3(1)$ & 1 & II & 12 & 0 & $3(3)$ & $9(7)$ \\
\hline III & 12 & 3 & 8 & $1(1)$ & III & 11 & 0 & $4(1)$ & $7(2)$ \\
\hline IV & 11 & 4 & 4 & $3(0)$ & IV & 7 & 0 & $1(1)$ & $6(5)$ \\
\hline V & 7 & 0 & $4(1)$ & $3(1)$ & - & - & - & - & - \\
\hline Total & 50 & $13(1)$ & $27(3)$ & $10(2)$ & & 40 & 1 & $12(6)$ & $27(15)$ \\
\hline
\end{tabular}

* Entre parênteses o número de casos com resultado normal à avaliação. "Avaliação na idade superior do nível" x "proporção de normais em cada nível" $r=0,92 \quad p=0,08$.

responderam ao nível esperado (Nível I); nas demais idades avaliadas, todas estavam adequadas aos níveis esperados II, III, IV e V, exceto uma criança. Esta não apresentou resposta condizente com o nível III cronológico, nem com o nível II quando se corrigiu a idade; entretanto, ao ser observada no nível IV, compreendeu as ordens simples propostas, mostrando-se adequada. Não foi portanto necessária, para a linguagem receptiva, a correção da idade para as crianças com idade cronológica igual ou superior a 4 meses.

Para a linguagem expressiva, considerando-se a idade cronológica, das 50 avaliações realizadas, $6(12 \%)$ mostraram desempenho para os níveis esperados. Foram realizadas 40 correções de idade (Tabela 2). Cinco (12,5\%) já estavam normais na avaliação sem correção da idade, 16 (40\%) adequaramse ao nível esperado (normalizaram) e 19 (47,5\%) ainda mostraram-se atrasadas. Das 20 crianças, 6 (30\%) mostraram-se sempre aquém dos níveis esperados e assim permaneceram, mesmo corrigindo- se a idade, 7 (35\%) normalizaram ao se corrigir a idade, 4 das quais também apresentaram um dos níveis normal para a idade cronológica. Sete (35\%) apresentaram variações nas respostas, ora mantendo-se atrasadas, ora normalizando ao se corrigirem as idades. Comparando os achados entre as idades corrigidas e cronológicas (Figs 1e 2), houve diferença estatística significante no nível II $(p=0,04)$ e IV $(p=0,002)$ com maior proporção de crianças normais com idade corrigida, e não houve nos níveis I e III $(p>0,05)$.

Na Tabela 3 estão distribuídos os casos de acordo com o período da avaliação por nível. O coeficiente de correlação entre as variáveis: "avaliação na idade superior do nível" e "proporção de normais em cada nível" foi $r=0,92$ indicando correlação positiva e não significativa $(p=0,08)$. Ainda para a idade corrigida, o coeficiente de correlação entre "avaliação na idade mediana do nível" e a proporção de normais em cada nível resultou $r=-0,93$ que indica correlação negativa e não significativa $(p=0,06)$.

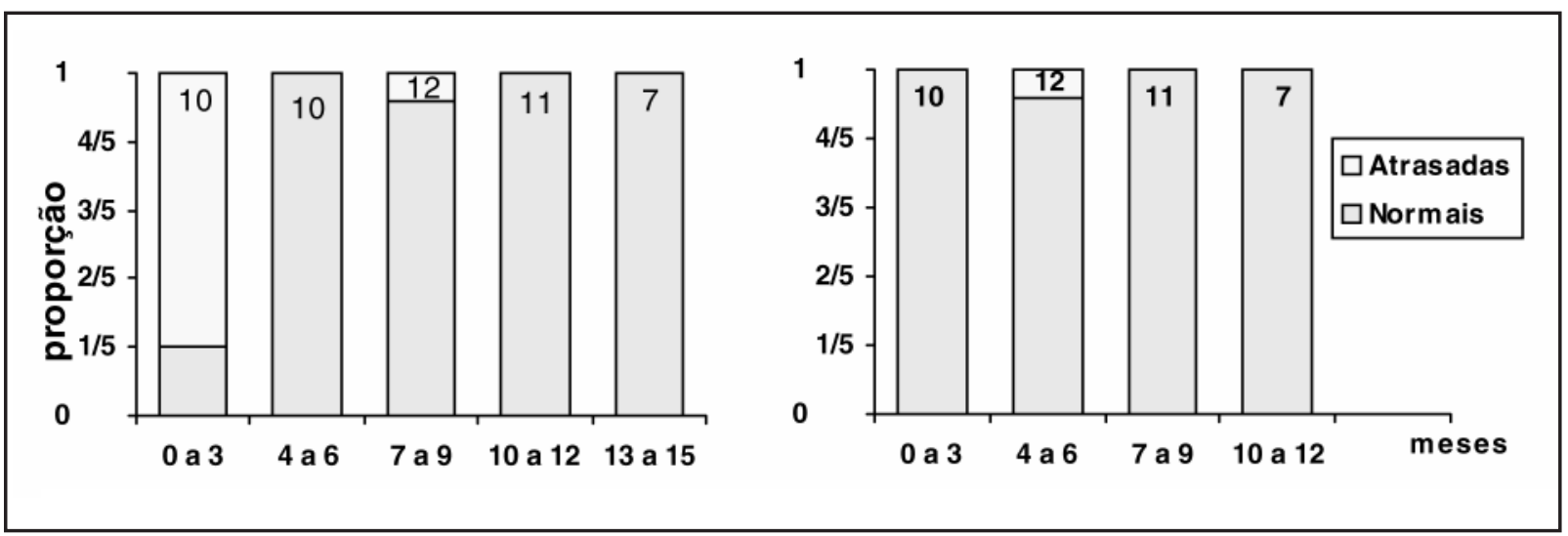

Fig 1. Proporção de normais e atrasadas para idade cronológica (esquerda) e corrigida (direita) quanto à linguagem receptiva. No topo, em cada grupo, o valor de $N$. 


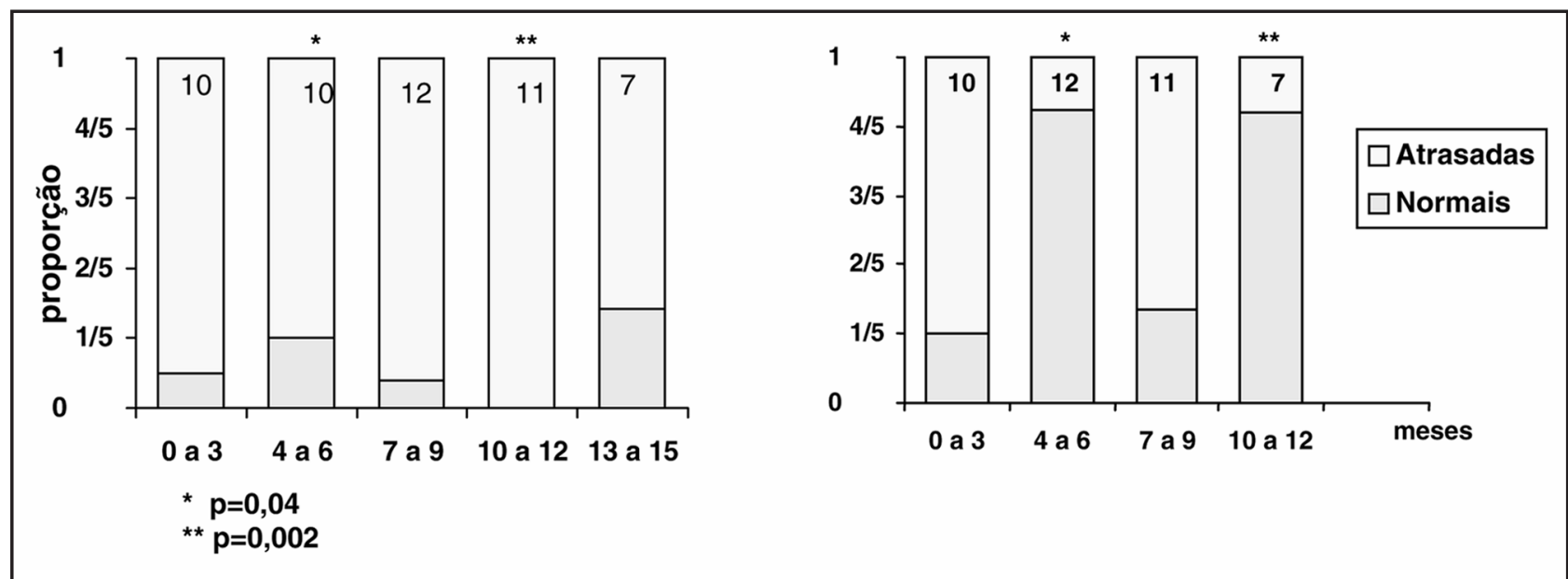

Fig 2. Proporção de normais e atrasadas para idade cronológica (esquerda) e corrigida (direita) quanto à linguagem expressiva. No topo, em cada grupo, o valor de $N$.

Comparando-se os grupos segundo a condição de AIG ou PIG, com a ressalva de que o $\mathrm{N}$ foi pequeno, considerando-se a idade cronológica: para a linguagem expressiva, observou-se uma criança normal das 11 PIG, e 5 normais das 9 AIG $(p<0,05)$. A comparação entre os níveis não evidenciou diferenças significativas. Corrigindo-se a idade, não houve diferença entre os grupos em nível de 0,05 comparando-se os dois grupos, se desempenho normal ou atrasado, em seu conjunto ou em cada nível considerado.

\section{DISCUSSÃO}

A primeira questão a ser considerada no presente trabalho é a da metodologia utilizada. A linguagem tem sido avaliada no contexto do estudo do desenvolvimento em outros domínios, e, neste sentido, mais recentemente, sua avaliação foi padronizada para crianças brasileiras ${ }^{16}$. Com a possibilidade de enfoque exclusivo na linguagem, em função da especialidade, Costa et al. ${ }^{11}$ apresentaram um roteiro para diagnóstico e acompanhamento de neonatos pré-termo que se completa, na atuação clínica, por um prontuário com os dados de identificação, antecedentes constitucionais pré, peri e pós-natais, e síntese de fatores de alto risco auditivo. Em alguns aspectos, a proposta de Costa et al. ${ }^{11}$, aqui utilizada, encontra similaridade em literatura posterior, como Stark e Goffman ${ }^{17}$. Ambos descrevem as etapas para o acompanhamento das aquisições e desenvolvimento da linguagem, e se referem à divisão em período pré-fala, que abrange os comportamentos perceptivos e produtivos da fala, e período lingüístico, que inclui compreensão e produção da linguagem através da fala, representatividade da linguagem através de jogos simbólicos, e do desenvolvimento do vocabulário e combinação de palavras. Há, entretanto, diferença na idade proposta para o limite do período prélingüístico, sendo 9 meses para Costa et al. ${ }^{11}$ e 18 meses para Stark e Goffman ${ }^{17}$. No presente trabalho, optamos pela adaptação deste roteiro em função da sua maior proximidade com a rotina na prática clínica.

Assim, a partir dessa técnica de investigação, em relação à avaliação da recepção da linguagem, não foram observados no presente trabalho atrasos relevantes, exceto para os 3 primeiros meses de idade cronológica. Embora as crianças desta pesquisa, entre 2 e 3 meses de idade cronológica, tenham demonstrado atraso quanto a reagir a vozes familiares, estes desvios foram superados entre os três primeiros meses de idade corrigida e, já ao redor dos quatro meses de idade cronológica, pode-se dizer "alcançaram" seus similares a termo quanto à recepção lingüística.

Quanto a respostas a vozes familiares nas idades cronológicas de 4 a 6 meses, na presente casuística todas as crianças reagiram à voz da mãe, apresentando comportamento esperado para este nível. Azevedo, em duas casuísticas ${ }^{18,19}$, encontrou freqüência menor, $43 \%$ e $38 \%$ respectivamente, de respostas esperadas, para a faixa etária de 3 a 6 meses, sendo esta amostra, entretanto, com idade inicial um mês inferior a da presente pesquisa, não equivalente ao Nível II aqui estudado. Nas demais provas para a linguagem receptiva, todas as crianças corresponderam aos níveis III a $\mathrm{V}$ espera- 
dos, exceto uma no nível III que, entretanto, apresentou-se normal nas avaliações subseqüentes.

Os resultados da presente casuística evidenciaram portanto a idade cronológica de 4 meses de equiparação para a linguagem receptiva, entre lactentes pré-termo e de termo, sendo esta idade inferior à observada por Bassetto ${ }^{20}, 12$ meses, em estudo de lactentes também considerados hígidos, da cidade de São Paulo. A diferença observada atribui-se à particularidades na metodologia utilizada, como critérios de normalidade neurológica, sendo considerado no presente trabalho, além do exame neurológico, a ultra-sonografia neonatal.

Em relação aos comportamentos pré-lingüísticos e lingüísticos expressivos, no presente estudo, as crianças apresentaram-se atrasadas em algum ponto desde o nível I ao V, tanto para a idade cronológica como corrigida. Corrigindo-se a idade, os atrasos ainda permaneceram no nível I pré-lingüístico e nível III lingüístico, início do desenvolvimento lingüístico. Os atrasos observados no nível I para a linguagem expressiva podem estar relacionados com o fato de que as avaliações ocorreram em idades muito precoces. Os atrasos observados no nível III podem estar relacionados, por exemplo, com o período em que as crianças começam a se preparar para entrar no mundo lingüístico, e este atraso lingüístico expressivo torna-se mais evidente pela própria troca de "exigência" comunicativa que o lactente já possui com o seu meio ambiente.

Para as funções corticais superiores, como a linguagem, a participação dos fatores biológicos, ambientais e sociais na sua formação, é indiscutível. Seria de se esperar, em relação à maturidade biológica, que ao corrigirmos as idades, ocorresse uma tendência crescente na criança pré-termo para adquirir a linguagem, em relação à de termo. Esta correlação não se observou na presente casuísti$c a$, ocorrendo alta freqüência de normalidade nos níveis II, que se reduz no nível III e volta a crescer no nível IV. As crianças observadas no período entre 4 a 6 meses de idade cronológica vocalizavam menos, enquanto que, com idade corrigida para a mesma faixa etária os comportamentos adequaram-se quanto à emergência para a expressão de sons vocálicos. As crianças avaliadas entre 7 a 9 meses de idade cronológica emitiram sons predominantemente vocálicos, e essa ocorrência de vocalizações permaneceu nesta mesma faixa etária para a idade corrigida. Estes achados sugerem que neste período não ocorreu a emergência do balbucio, en- contrando-se ausente a repetição de seqüências de consoantes e vogais, sendo o típico exemplo do "ma-ma-ma", "dadada", "papapa". Considerandose a idade cronológica, nenhuma criança avaliada entre 10 e 12 meses, período caracterizado pelo início da fala lingüística, emitia as primeiras duas ou três palavras com intenção de comunicação, constatando atraso quanto ao desempenho da expressão verbal para a idade cronológica nesta faixa etária. Corrigindo-se as idades, neste mesmo período, não se observou atraso na quase totalidade dos casos. Para a faixa etária entre 13 a 15 meses de idade cronológica observou-se que de um total de 7 crianças avaliadas, apenas 2 expressavam entre 6 a 10 palavras com significado nomeando objetos, onomatopéias e situações.

A dimensão da influência ambiental no processo do atraso tem recebido atenção por parte de pesquisadores no sentido de se buscarem dados conclusivos a respeito de fatores como o período de hospitalização do recém-nascido, suporte familiar e do psicológico materno frente ao impacto do nascimento pré-termo ${ }^{7,21-23}$. Crawford ${ }^{24}$ observou que aos seis meses de idade cronológica, as crianças prétermo comportavam-se de um modo diferente das crianças de nascimento a termo. As crianças vocalizavam menos, brincavam menos, e eram mais irritáveis que as a termo, porém olhavam mais para os objetos e ao redor do seu ambiente. Essas diferenças, com exceção da vocalização, desapareciam por volta dos 14 meses. No entanto, as crianças prétermo nunca vocalizavam tão freqüentemente quanto as nascidas a termo. Bassetto ${ }^{20}$ avaliou crianças pré-termo sem lesões no sistema nervoso central aos 12 meses de idade cronológica, e observou que o desempenho da expressão oral foi significativamente inferior ao das crianças nascidas a termo.

Na presente pesquisa, a relativa superioridade para o desempenho quando se corrige a idade nos níveis II e IV sugerem que os fatores biológicos são significativos em algumas fases do desenvolvimento inicial, e, por outro lado, um desempenho normal com o uso da idade cronológica representaria algum grau de influência do meio ambiente e, essas influências se tornariam mais significativas com o desenvolvimento subseqüente, como sucedeu também com os achados de Siegel ${ }^{2}$.

Assim, diante das evidências de que, a aquisição e desenvolvimento da linguagem encontram-se defasadas, mesmo que o bebê pré-termo apresente bom desenvolvimento motor grosseiro, e, mesmo 
que se demonstre que tal defasagem seja um ritmo próprio do pré-termo, que mais tarde se igualará à criança de termo, entendemos que um auxílio no sentido de estimular os processos integrativos, de funções corticais superiores, com uso do verbal, se faz necessário desde o período pré-lingüístico. Este procedimento constitui-se em medida preventiva para futuros problemas na aprendizagem dos códigos de comunicação, principalmente se raciocinarmos segundo as etapas do processo da linguagem propostas por Perissinoto ${ }^{25}$ aquisição, desenvolvimento e abrangência.

Além do enfoque preventivo pela condição de pré-termo, apesar dos achados da reduzida amostra na presente pesquisa, entendemos que atenção especial é necessária para o bebê PIG. Houve apenas 1 criança normal das 11 PIG, quando se considerou a idade cronológica, enquanto que 5 das 9 AIG foram normais, indicando uma tendência para a associação entre normalidade sem a correção da idade e a condição de AIG. Com a correção da idade não houve diferença entre PIG e AIG, sugerindo que, na condição de PIG, com a correção poderia não se detectar atrasos e postergar medidas de prevenção, com prejuízo para a criança. Os estudos mais recentes compilados da literatura focalizam em sua maioria os fatores de risco e desempenho aquém dos seus pares em idades maiores ${ }^{8-11}$.

No Brasil, Oliveira et al. ${ }^{26}$, utilizando a Escala Early Language Milestone traduzida para o português ${ }^{27}$, compararam recém-nascidos de termo PIG a pré-termo AIG. Ambos os grupos apresentaram até o sexto mês produção equivalente para a idade cronológica. Este achado difere do observado na presente amostra, que já apresentava atraso, mesmo nas idades corrigidas para o nível I e II. Os itens aqui considerados levam em conta exclusivamente emissões vocálicas até o sexto mês, enquanto que no ELM são citados o sorriso e vibração dos lábios como primeiras manifestações. No $9^{\circ}$. mês os itens da ELM são os mesmos aqui utilizados e observase similaridade entre os dois trabalhos na menor produção durante o período da emergência do balbucio, embora se tenha encontrado pelo ELM que tais achados também ocorreram em crianças de termo AIG. A interpretação de resultados em estudos sobre linguagem, é raramente comparável em função dos diferentes métodos e delineamentos utilizados.

Os achados na presente pesquisa são relevantes, uma vez que um grupo pré-termo PIG ou AIG, com exame neurológico e do desenvolvimento neuromotor normais, evidenciou atraso específico de linguagem expressiva desde a sua aquisição, mesmo com idade corrigida, quando comparados a um referencial obtido da experiência clínica, presente no roteiro de Costa et al. ${ }^{11}$.

Em conclusão, os resultados obtidos, a partir do roteiro de Costa et al. ${ }^{11}$, em uma amostra de crianças nascidas pré-termo sem sinais clínicos de lesão cerebral, permitem-nos afirmar que ${ }^{28:} 1$ - Não foi necessária a correção da idade para a avaliação da linguagem receptiva verbal. 2 - Em relação à linguagem expressiva, mesmo nas idades corrigidas, houve atrasos tanto no nível pré-lingüístico como no lingüístico. 3 - Nas idades corrigidas para 6 meses e 12 meses a adequação ao nível esperado foi relevante. 4 - Os resultados para linguagem expressiva aos 9 meses sugerem que esta amostra não apresentou alcance satisfatório do período lingüístico nesta idade. 5 - A avaliação, considerando-se a idade cronológica, apresentou tendência a predomínio de crianças nascidas AIG entre aquelas com desempenho normal, e o inverso ocorreu para as crianças PIG. A idade corrigida não distinguiu os dois grupos.

A observação do desenvolvimento lingüístico expressivo de crianças pré-termo forneceu subsidio para as proposições: Intensificar a vigilância da aquisição e desenvolvimento da linguagem aos 6 e 12 meses de idade corrigida. As correções para o grau da prematuridade sejam realizadas à partir do quarto mês de vida (nível II), pois alguns dos resultados atrasados obtidos no nível I para a idade corrigida podem ter ocorrido em função das idades muito precoces. Enfatizar ainda mais a necessidade de cuidados maiores e atenção ao seguimento do bebê prematuro, principalmente se for PIG, mesmo com exame neurológico e do desenvolvimento neuromotor normais. Conduzir a orientação às mães, estimulação da linguagem e encaminhamentos que se fizerem necessários o mais cedo possível, em função do número de casos observados com atraso. Reavaliar cada caso para controle evolutivo até completar o período lingüístico, mesmo com a linguagem normal ou normalizada em um dado momento do primeiro ano.

Agradecimento - A Profa. Dra. Jacy Perissinoto, Profa. Adjunto da Disciplina de Distúrbios da Comunicação Humana do Departamento de Fonoaudiologia da Universidade Federal de São Paulo- UNIFESP, pela coorientação que proporcionou com conhecimento específico e ampla experiência na área. 


\section{REFERÊNCIAS}

1. Babson SG. Growth of low-birth-weight infants. J Pediatr 1970;77:11-18.

2. Siegel LS. Correction for prematurity and its consequences for the assessment of the very low birth weight infant. Child Dev 1983;54:1176-1188.

3. Ungerer JA, Sigman M. Developmental lags in preterm infants from one to three years of age. Child Dev 1983;54:1217-1228.

4. Allen MC, Alexander GR. Gross motor milestones in preterm infants: correction for degree of prematurity. J Pediatr 1990;116:955-959.

5. Lems W, Hopkins B, Samson JF. Mental and motor development in preterm infants: the issue of corrected age. Early Human Dev 1993;34:113-123.

6. Matilainen R. The value of correction for age in the assessment of prematurely born children. Early Human Dev 1987;15:257-264.

7. Normand M, Vaivre-Douret L, Delfosse MJ. Language and motor development in preterm children: some questions. Child: Care, Health Dev 1994;21:119-133.

8. Gutbrod T, Wolke D, Soehne B, Ohrt B, Riegel K. Effects of gestation and birth weight on the growth and development of very low birthweight small for gestational age infants: a matched group comparison. Arch Dis Child Fetal Neonatal 2000;82:208-214.

9. Monset-Couchard M, de Bethmann O, Kastler B. Mid-and long-term outcome of 166 premature infants weighing less than $1000 \mathrm{~g}$ at birth, all small for gestational age. Biol Neonate 2002;81:244-254.

10. Rooney R, Hay D, Levy F. Small for gestational age as a predictor of behavioral and learning problems in twins. Twin Research 2003;6:46-54.

11. Costa SA, Gentile C, Gomes Pinto ALP, Perissinoto J, Pedromônico MRM, Azevedo MF Roteiro de diagnóstico e acompanhamento do desenvolvimento de crianças de 0 a 36 meses de idade. Pró-Fono - Revista de Atualização Científica 1992;4:9-15.

12. Capurro H. Método clínico para diagnosticar la edad gestacional del recién nacido. Tese (Doutorado), Facultad de Med. Universidad de La Rep Montevideo, 1973.

13. Ballard JL, Novak KK, Driver M. A simplified score for assessment of fetal maturation of newly born infants. J Pediatr 1979;95:769-774.

14. Ballard JL, Khoury JC, Wedig K, et al. New Ballard Score, expanded to include extremely premature infants. J Pediatr 1991;119:417-423.

15. Sala MM Estudo do crescimento intrauterino na segunda metade da gestação: determinação dos percentis $10,25,50,75$ e, 90 do peso placentário, índice placentário, peso e estatura fetal.Tese(Livre-Docência), Faculdade de Medicina de Ribeirão Preto, Universidade de São Paulo. Ribeirão Preto, 1977.

16. Pinto EB. O desenvolvimento do comportamento da criança no primeiro ano de vida: padronização de uma escala para a avaliação e o acompanhamento. São Paulo: Casa do Psicólogo, 1997.

17. Stark RE, Goffman L. Development of speech and language. In BERG BO (ED) Principles of child neurology. New York: McGraw-Hill; 1996: 383-396.

18. Azevedo MF. Avaliação e acompanhamento audiológico de neonatos de risco. Acta WHO 1991;19:107-115.

19. Azevedo MF. Desenvolvimento auditivo de crianças normais e de alto risco: estudo comparativo das respostas comportamentais a estímulos sonoros. Tese (Doutorado), Escola Paulista de Medicina. São Paulo. 1993

20. Bassetto MCA. Crianças nascidas pré-termo e de baixo peso: estudo de aspectos auditivos e lingüísticos. Tese (Mestrado), Universidade Federal de São Paulo, São Paulo, 1994.

21. Werner EE, Smith RS. Kauai's children come of age. Honolulu: University Press of Hawai, 1977. In Rocissano L. Yatchmink Y. (EDS) Language skil and interactive patterns in prematurely born toddlers. Child Dev 1983;54:1229-1241.

22. Linhares MBM, Carvalho AEV, Padovani FHP, Bordin MBM, Martins IMB, Martinez FE. A compreensão do fator de risco da prematuridade sob a ótica desenvolvimental In: Vulnerabilidade e proteção: indicadores na trajetória de desenvolvimento do escolar ed.São Paulo: Casa do Psicólogo, 2002.

23. Martins IMB, Linhares MBM, Martinez FE. Análise das interações entre mães e crianças pré-escolares nascidas pré-termo e de baixo peso em situação de observação estruturada de brincar. Arq Neuropsiquiatr 2000;(Supl 1):S12.

24. Crawford JW. Mother infant interaction in premature and full-term infants. Child Dev 1982;53:957-962

25. Perissinoto J. Psicose e neurose em crianças: estudo quantitativo do desenvolvimento motor e da linguagem. Tese (Doutorado), Escola Paulista de Medicina. São Paulo. 1992.

26. Oliveira LN, Lima MCMP, Gonçalves VMG. Acompanhamento de lactentes com baixo peso ao nascimento: aquisição de linguagem. Arq Neuropsiquiatr 2003;61:802-807.

27. Lima MCMP. Avaliação da fala de lactentes no período pré-liguístico: uma proposta para triagem de problemas auditivos. Tese, Universidade Estadual de Campinas (UNICAMP). Campinas. 1997.

28. Jorge MRP. Avaliações de alguns aspectos da aquisição e desenvolvimento da linguagem de crianças nascidas pré-termo considerandose as idades cronológica e corrigida. Dissertação de Mestrado, Universidade de São Paulo, Ribeirão Preto, 2003. 